\title{
Wind direction extraction from synthetic aperture radar (SAR) using wavelet transform technique
}

\begin{abstract}
Backscatter of RadarSat-1 SAR images for incidence angles between $20^{\circ}$ to $70^{\circ}$ at an ocean area is caused by Bragg scattering. The Bragg-scale waves created based on the response of the ocean to the wind stress. Therefore, wind direction can be retrieved in radar images from the orientation of the wind streaks that visible in the images. However, the variation of the incidence angle can cause geometric distortion and variation in scattering components. SAR images for Wide-3 and Extended-High 6 modes have produced wind direction which similar as recorded in AWAC data with correlation are $r=0.94$ and $r=0.98$ respectively. The wind blows for both images is correspond during North East Monsoon (September to March) with wind speed about $0.9 \mathrm{~m} / \mathrm{s}$. However, weak correlation is exhibits for Standard-2 ( $\mathrm{r}=0.29)$, it may due to wide range of incidence angle that slow wind speed become invisible to the wavelet formation. From the result, it shows that Extended High-6 mode is considered the best mode to make use for extracting wind direction with high correlated with AWAC data. This is also confirmed that large incident angle with small range angle provides more information on wind direction, as Extended High-6 having the largest incidence angle among the two modes. As conclusion, the SAR backscatter is closely affected by the incidences angles, and this can provide on wind direction information for wind studies.
\end{abstract}

Keyword: Synthetic aperture radar (SAR); Wind direction; Wavelet transform; Incidence angle 